\title{
Excitonic mobility edge and ultra-short photoluminescence decay time in $\mathbf{n}$-type
} GaAsN

\author{
F. Eßer, ${ }^{1,2, a)}$ S. Winnerl, ${ }^{1}$ A. Patanè, ${ }^{3}$ M. Helm, ${ }^{1,2}$ and H. Schneider ${ }^{1}$ \\ 1) Helmholtz-Zentrum Dresden-Rossendorf, Institute of Ion Beam Physics \\ and Materials Research, Bautzner Landstraße 400, 01328 Dresden, \\ Germany \\ 2) Technische Universität Dresden, 01062 Dresden, Germany. \\ 3) School of Physics and Astronomy, University of Nottingham, \\ NGr7 2RD United Kingdom
}

(Dated: 23 September 2016)

We use time-resolved photoluminescence (PL) spectroscopy to study the recombination dynamics in Si-doped GaAsN semiconductor alloys with a nitrogen content up to $0.2 \%$. The PL decay is predominantly monoexponential and exhibits a strong energy dispersion. We find ultra-short decay times on the high-energy side and long decay times on the low-energy side of the photoluminescence spectrum. This asymmetry can be explained by the existence of an additional non-radiative energy transfer channel and is consistent with previous studies on intrinsic GaAsN epilayers. However, the determined maximum decay times of GaAsN:Si are significantly reduced in comparison to undoped GaAsN. The determined excitonic mobility edge energy constantly decreases with increasing $\mathrm{N}$ content, in agreement with the two-level band anticrossing model.

PACS numbers: 71.35.-y, 71.55.-i, 78.20.-e, 78.47.jd, 78.55.Hx

a) Electronic mail: faina.esser@posteo.de 
Dilute nitrides like (In) $\mathrm{GaAs}_{1-x} \mathrm{~N}_{x}$ have attracted considerable attention in the last decades because of their band gap tunability ${ }^{1,2}$ in the telecommunication region and beyond ${ }^{3}$. The first $\operatorname{In}_{y} \mathrm{Ga}_{1-y} \mathrm{As}_{1-x} \mathrm{~N}_{x}$-based laser with an emission wavelength of $1.3 \mu \mathrm{m}$ was demonstrated in 2000 by Livshits et al. ${ }^{4}$. Vertical cavity surface emitting lasers ${ }^{5,6}$ and edge-emitting devices $^{7}$ for $1.5 \mu \mathrm{m}$ have been presented in subsequent years. Besides their technological potential, dilute nitrides feature unusual fundamental properties like e.g. a giant band gap reduction $^{1}$ with $\mathrm{N}$ content, an unusual pressure and temperature dependence of the electronic properties ${ }^{8-10}$, highly non-parabolic conduction bands ${ }^{11}$, and the difficulty in doping GaAsN with Si-dopants due to the formation of Si-N complexes ${ }^{12}$. Different methods have been applied on the system GaAsN in order to determine the electron effective mass. A decrease $^{13}$, an increase ${ }^{14-16}$ and a non-monotonic dependence ${ }^{17}$ of the electron effective mass with increasing $\mathrm{N}$ content were observed. Using cyclotron resonance absorption spectroscopy, we found a moderate increase of the electron effective mass with increasing $\mathrm{N}$ content ${ }^{18}$. Our result is in excellent agreement with calculations based on the two-level band anticrossing (BAC) model $^{9}$. Thus we conclude that the different sensitivity of the used methods to carrier localization effects is responsible for contradicting results.

A significant size and electronegativity difference between nitrogen and arsenic atoms causes a non-uniform $\mathrm{N}$ distribution, which creates localization potentials and increases the disorder of the system. Photoluminescence (PL) is particularly sensitive to disorder effects like N-induced localization centers. Compositional fluctuations ${ }^{19-21}$ create regions with a locally reduced band gap and lead to PL emission at lower energies, similarly to quantum $\operatorname{dots}^{20,22}$. Localized excitons dominate the low-temperature $\mathrm{PL}^{19,22,23}$ and exhibit strongly energy-dependent PL decay times ${ }^{19,24}$. Since most telecommunication applications require $\mathrm{n}$ (or p)-type doping, it is highly desirable to understand the impact of a typical dopant like silicon on the recombination dynamics of GaAsN.

We apply a systematic and temperature dependent time-resolved PL study on Si-doped $\operatorname{GaAs}_{1-x} \mathrm{~N}_{x}$ epilayers with $x=0 \%-0.2 \%$. The GaAsN:Si layers were grown by molecular beam epitaxy at a reduced temperature of $500^{\circ} \mathrm{C}$ with a thickness of $1 \mu \mathrm{m}$. The $\mathrm{N}$ content was monitored by $\mathrm{x}$-ray diffraction and the n-doping is nominally $1 \times 10^{17} \mathrm{~cm}^{-3}$. More details on the sample series can be found in Refs. ${ }^{18,25}$. For the time-resolved PL study, the samples were illuminated by a picosecond titanium-sapphire laser (Spectra Physics: Tsunami 3960) with a photon energy of $1.61 \mathrm{eV}$. The detection unit consists of a Bruker Chromex 250is/is 


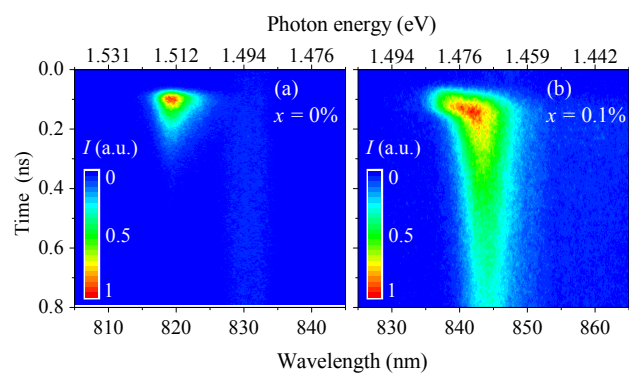

FIG. 1. Streak camera images of the PL decay from $\operatorname{GaAs}_{1-x} \mathrm{~N}_{x}$ :Si with (a) $x=0 \%$ and (b) $x=0.1 \%$ and an average power density of $9.5 \mathrm{~W} / \mathrm{cm}^{2}$ at $10 \mathrm{~K}$. The intensities are normalized for better comparison between samples.

spectrograph, a Hamamatsu C5680-27 streak camera and a Hamamatsu ORCA-ER C474295 charge-coupled device. The sample was cooled in a He cryostat enabling measurements at different temperatures.

Fig. 1 shows streak camera images of the time-resolved PL of $\operatorname{GaAs}_{1-x} \mathrm{~N}_{x}: \mathrm{Si}$ with $x=0 \%$ and $x=0.1 \%$. The PL decay of the $\mathrm{N}$ containing sample in Fig. 1 (b) exhibits a strong energy dispersion in comparison to the reference sample in Fig. 1 (a). We obtain ultrashort decay times on the high energy side and long decay times on the low-energy side of the spectrum. Similar observations were made in intrinsic (In)GaAsN epilayers ${ }^{26-28}$ and quantum-wells ${ }^{24,29}$.

In order to study the origin of this energy dispersion, we investigate line profiles of the PL decay at given photon energies. Three neighboring low-temperature decay line profiles are shown in Fig. 2 (a) - (c). The underlying time-resolved PL measurement is taken at $5 \mathrm{~K}$ and $9.5 \mathrm{~W} / \mathrm{cm}^{2}$. The decay dynamics is characterized by a fit function which is a sum of a monoexponential decay and a constant offset, as shown with pink curves. The offset accounts for recombination processes with ultra-long decay times, which originate from efficiently trapped carriers, as observed e.g. in n-type InGaAs/GaAs quantum $\operatorname{dots}^{30}$. The monoexponential function mainly describes the decay of localized excitons and trions. The corresponding localization centers were attributed to compositional fluctuations in $\mathrm{N}$ content ${ }^{19-21}$ as observed by e.g. transmission electron microscopy ${ }^{20}$. Localized excitons have different discrete energetic levels below the band minimum of free excitons, induced by the corresponding localization potentials. The observation of sharp distinct peaks in near-field microscopy ${ }^{20,21}$ of (In)GaAsN supports this interpretation. A very short time constant can be found in the first 


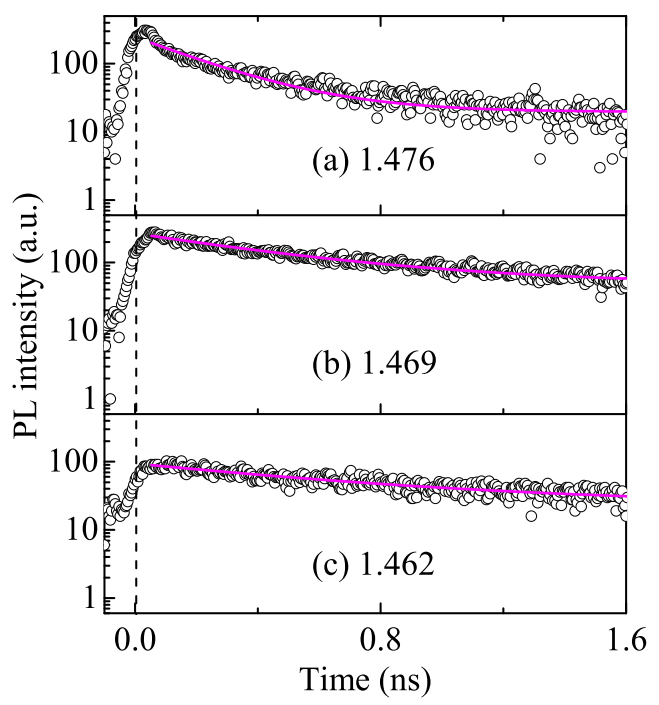

FIG. 2. (a) - (c) PL decay line profiles of different energy positions of time-resolved PL in $\operatorname{GaAs}_{1-x} \mathrm{~N}_{x}$ :Si with $x=0.1 \%$ at $5 \mathrm{~K}$ and $9.5 \mathrm{~W} / \mathrm{cm}^{2}$. Circles represent experimental data points and pink lines illustrate monoexponential fit functions.

100 ps in Fig. 2 (a) shortly after laser excitation. This ultrafast PL decay, which is typical for the high energy side of the spectrum is related to carrier cooling ${ }^{31}$. Note that intraband scattering of photoexcited electrons occurs on an even shorter timescale, namely on a sub-ps scale $^{32}$. These fast parts of the dynamics are not within the scope of this study. A schematic representation of all radiative and non-radiative recombination and decay channels is given in Fig. 3. We use the monoexponential decay function after a certain thermalization time of 50 ps for our data analysis. The PL decay time of localized excitons decreases by a factor of 3.1 in a small energy interval of $14 \mathrm{meV}$.

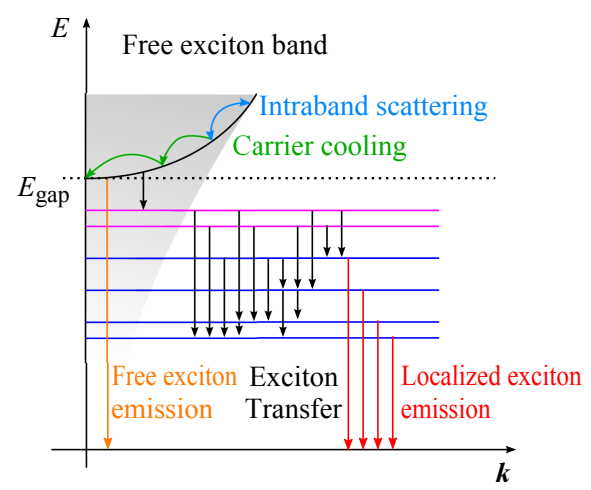

FIG. 3. Schematic representation of free and localized excitonic states and recombination processes after optical laser excitation of GaAsN. 
The resulting PL decay times of the different line profiles are shown in Fig. 4 in an energy range of $1.44 \mathrm{eV}-1.48 \mathrm{eV}$ by full circles. For a better overview, the time-integrated low-temperature PL spectrum of $\mathrm{GaAs}_{0.999} \mathrm{~N}_{0.001}$ :Si is also displayed (empty circles). Furthermore, the energy positions of the shown PL line profiles (see Fig. 2) are indicated with vertical arrows. The mentioned energy dispersion of the PL decay times $\tau(E)$ is very pronounced in this representation. It can only be explained by the existence of an additional non-radiative energy transfer channel. We study the mechanism of this energy dispersion in the following.

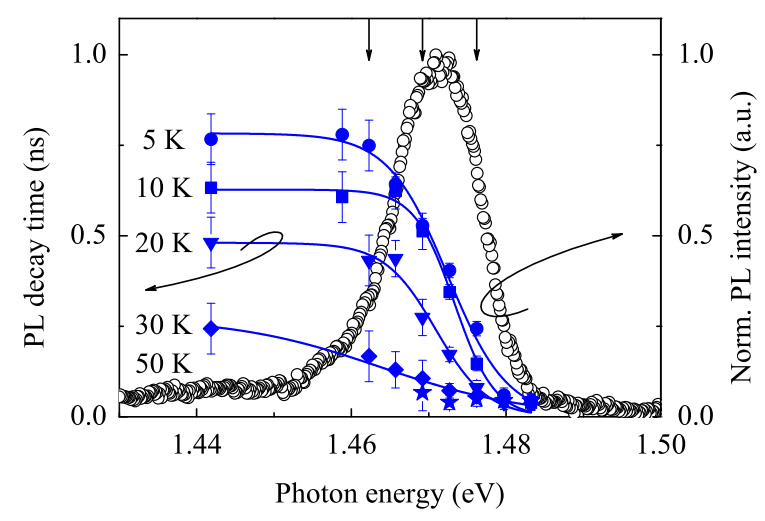

FIG. 4. Temperature dependence of the decay time dispersion. Full symbols represent PL decay times, which were taken at $5 \mathrm{~K}-50 \mathrm{~K}$. Solid lines correspond to a fit from Eq. (1). The normalized PL intensity of the $5 \mathrm{~K}$ spectra is shown for comparison (empty circles). The energy positions of the shown PL line profiles in Fig. 2 (a) - (c) are indicated by vertical arrows.

The energy dependent PL decay of localized excitons can be fitted by ${ }^{26,33}$

$$
\tau(E)=\frac{\tau_{R}}{1+\exp \left(\alpha\left(E-E_{m}\right)\right)}
$$

with a maximum decay time $\tau_{R}$, an energy $E_{m}$ corresponding to the so-called mobility edge (see below) and an energy scaling factor $\alpha$ with $1 / \alpha$ in the order of $2.59 \mathrm{meV}-9.55 \mathrm{meV}$. According to Eq. (1), excitons with an energy $E$ can recombine radiatively or be transferred non-radiatively towards lower energy states. The non-radiative transfer can be regarded as an exciton hopping process. After optical excitation, excitons preferentially hop several times to lower energies before they recombine radiatively ${ }^{34}$. Consequently, the non-radiative transfer dominates on the high-energy side of the PL spectrum, which corresponds to all energies $E>E_{m}$. The observed radiative transitions are either delocalized or slightly localized excitons, which can be easily transferred out of their sites ${ }^{26}$. Thus, they have extremely 
short decay times: e.g. $\tau(1.476 \mathrm{eV})=0.24 \mathrm{~ns}$ for $5 \mathrm{~K}$. On the other hand, most transitions are radiative below $E_{m}$ and correspond to strongly localized excitons and trions with longer decay times. For $\mathrm{GaAs}_{0.999} \mathrm{~N}_{0.001}$ :Si, slow recombination processes dominate for $E<1.47 \mathrm{eV}$. Thus, the so-called mobility edge ${ }^{33} E_{m}$ corresponds to an energy with equal recombination and transfer rates ${ }^{26}$. Furthermore, the non-radiative transfer rate scales exponentially with the energy difference. An overview of the fitting results is provided in Tab. I.

\begin{tabular}{lccccc|c}
\hline \hline & & $x=0.1 \%$ & $x=0.2 \%$ \\
\cline { 2 - 6 } & & & & & \\
Temperature & $5 \mathrm{~K}$ & $10 \mathrm{~K}$ & $15 \mathrm{~K}$ & $20 \mathrm{~K}$ & $30 \mathrm{~K}$ & $10 \mathrm{~K}$ \\
$E_{m}(\mathrm{eV})$ & 1.472 & 1.473 & 1.472 & 1.471 & 1.464 & 1.445 \\
$\tau_{R}(\mathrm{~ns})$ & 0.78 & 0.63 & 0.63 & 0.48 & 0.27 & 0.44 \\
$1 / \alpha(\mathrm{meV})$ & 3.8 & 2.6 & 2.8 & 3.3 & 9.5 & 3.6 \\
\hline \hline
\end{tabular}

TABLE I. Fit results for the decay time dispersion with Eq. (1) for $\operatorname{GaAs}_{1-x} \mathrm{~N}_{x}$ :Si with $x=0.1 \%$ and $x=0.2 \%$.

The temperature dependent PL decay times are presented in Fig. 4 by full symbols. Solid lines represent fits with Eq. (1). It is evident that the energy dispersion of the PL decay rapidly decreases at low energies in the investigated temperature range, similarly to the temperature behavior of undoped (In) GaAsN $26,28,29$. Increasing the temperature enhances the diffusion of excitons towards localized states with lower energies. Furthermore, some localized excitons are ionized into delocalized states. As expected, both reduce the PL decay time $\tau_{R}$ of localized excitons (see Tab. I). The decay time depends only weakly on the illumination power up to $100 \mathrm{~mW}$ and decreases only at high powers due to a temperature effect. In contrast to $\tau_{R}$, no significant change at temperatures below $30 \mathrm{~K}$ is observed for $\alpha$ and $E_{m}$. Representative temperature dependent spectra of Si-doped GaAsN are also discussed in the Supplemental material.

Localized excitons of $\mathrm{GaAs}_{1-x} \mathrm{~N}_{x}:$ Si with $x=0.2 \%$ can be similarly described by Eq. (1), as shown in Fig. 5. In analogy to Fig. 4, energy dependent decay times and fits are presented with time-integrated PL curves. The reference sample has negligible dispersion in the investigated energy range. The decay times of the free exciton are almost constant with 73.9 ps $\leq \tau \leq 75.4$ ps, as shown in Fig. 5 (a). These decay times, being significantly shorter than the ns times observed in high-purity $\mathrm{GaAs}^{35,36}$, are consistent with PL studies on 


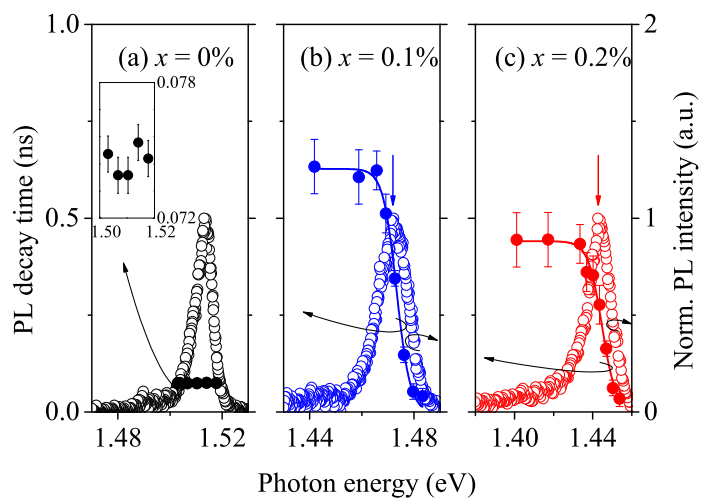

FIG. 5. Energy dispersion of the decay time (full circles) in $\operatorname{GaAs}_{1-x} \mathrm{~N}_{x}$ :Si with (a) $x=0 \%$, (b) $x=0.1 \%$ and (c) $0.2 \%$. Solid lines correspond to a fit with Eq. (1). Empty circles represent normalized PL spectra. The time-resolved PL is performed at $10 \mathrm{~K}$ with $9.5 \mathrm{~W} / \mathrm{cm}^{2}$. The inset zooms in on the negligible energy dispersion of the reference in (a). Vertical arrows indicate the determined energies of $E_{m}$.

GaAs grown at different temperatures ${ }^{37}$. The reference sample has a better lattice quality in comparison to nitrogen containing samples. Thus, exciton hopping is insignificant and the PL decay exhibits practically no energy dispersion.

As mentioned before, the decay time dispersion is very pronounced in both $\mathrm{N}$ containing samples in Fig. 5 (b) - (c). Furthermore, the maximum decay time $\tau_{R}$ is reduced in the $x=0.2 \%$ sample in comparison to $x=0.1 \%$, as shown in Tab. I. This observation is attributed to a more efficient exciton transfer (hopping) because of an enhanced defect density in the $x=0.2 \%$ sample in comparison to the $x=0.1 \%$ sample. The excitonic mobility edge $E_{m}$ continuously decreases with the $\mathrm{N}$ content. The decrease is in excellent agreement with the decrease of the band gap energy within the two-level BAC model ${ }^{9}$, as shown in Fig. 6.

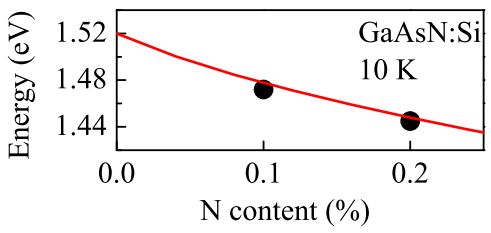

FIG. 6. Decrease of the excitonic mobility edge energy $E_{m}$ with increasing $\mathrm{N}$ content. Full circles correspond to the calculated values of $E_{m}$ for $10 \mathrm{~K}$ using Eq. (1). The solid line represents the decrease of the band gap energy of GaAsN according to the two-level BAC model ${ }^{9}$. 
A similar decay time asymmetry has previously been observed in intrinsic GaAsN epilayers $^{24,27}$. However, our determined maximum decay time values are significantly shorter (up to $\tau_{R} \approx 0.8 \mathrm{~ns}$ ) than the reported values of $\tau_{R} \approx 5-8 \mathrm{~ns}^{27}$ in the intrinsic material. It is well-known that n-type doping reduces the band-band recombination time, since it increases the product of the electron and hole densities

$$
n p=\left(n_{\mathrm{opt}}+n_{\mathrm{dop}}\right) p_{\mathrm{opt}}
$$

where $n_{\mathrm{opt}}$ and $p=p_{\mathrm{opt}}$ are the concentrations of optically injected electrons and holes, respectively, and $n_{\text {dop }}$ is the (electrically active) doping concentration. Nevertheless, in case of n-doped $\mathrm{GaAs}^{38,39}$ the impact of the doping is insignificant below $10^{18} \mathrm{~cm}^{-3}$. Furthermore, Si-doping leads to a higher contribution by non-radiative Shockley-Read-Hall and Auger recombination rates, as was observed in n-type GaAs ${ }^{40}$. Especially Shockley-Read-Hall recombinations significantly increase with the defect density. This behavior has a substantial impact on possible applications.

In summary, we performed temperature dependent time-resolved photoluminescence investigations on Si-doped GaAsN alloys with different nitrogen contents. Because of an additional non-radiative interexcitonic transfer channel, we found a strong energy dispersion of the photoluminescence decay times. The photoluminescence decay is predominantly non-radiative on the high-energy side of the spectrum. The radiative transitions are mainly delocalized and have ultra-short decay times in comparison to the low-energy part of the spectrum, which is characterized by radiative transitions of localized excitons and trions. An increase of the bath temperature reduces the decay times and thus the energy dispersion, in agreement with previous observations in intrinsic (In) GaAsN ${ }^{26,28,29}$. However, the determined maximum decay times of GaAsN:Si are significantly reduced in comparison to undoped GaAsN ${ }^{27}$. This can be explained by a higher contribution of Shockley-Read-Hall and possibly Auger recombinations in GaAsN:Si in comparison to undoped GaAsN. We determined the excitonic mobility edge energy as a function of the $\mathrm{N}$ content. The edge energy decreases with increasing $\mathrm{N}$ content, which is in excellent agreement with the two-level BAC model $^{9}$. 


\section{ACKNOWLEDGMENTS}

We gratefully acknowledge M. Hopkinson and coworkers for growing excellent samples.

\section{REFERENCES}

${ }^{1}$ M. Weyers et al., Jpn. J. of Appl. Phys. 31, L853 (1992).

${ }^{2}$ W. G. Bi and C. W. Tu, Appl. Phys. Lett. 70, 1608 (1997).

${ }^{3}$ A. Krier et al., Semicond. Sci. Technol. 27, 094009 (2012).

${ }^{4}$ D. A. Livshits et al., Electron. Lett. 36, 1381 (2000).

${ }^{5}$ G. Steinle et al., Electron. Lett. 37, 632 (2001).

${ }^{6}$ H. Riechert et al., Semicond. Sci. Tech. 17, 892 (2002).

${ }^{7}$ S. R. Bank et al., IEEE J. Quantum. Elect. 40, 656 (2004).

${ }^{8}$ P. Perlin et al., Appl. Phys. Lett. 73, 3703 (1998).

${ }^{9}$ W. Shan et al., Phys. Rev. Lett. 82, 1221 (1999).

${ }^{10}$ J. Endicott et al., Phys. Rev. B 72, 041306(R) (2005).

${ }^{11}$ J. Endicott et al., Phys. Rev. Lett. 91, 126802 (2003).

${ }^{12}$ K. M. Yu et al., Nat. Mater. 1, 185 (2002).

${ }^{13}$ D. L. Young et al., Appl. Phys. Lett. 82, 1236 (2003).

${ }^{14}$ P. N. Hai et al., Appl. Phys. Lett. 77, 1843 (2000).

${ }^{15}$ N. Mori et al., AIP Conf. Proc. 1399, 23 (2011).

${ }^{16}$ K. Alberi et al., Phys. Rev. Lett. 110, 156405 (2013).

${ }^{17}$ F. Masia et al., Phys. Rev. B 73, 073201 (2006).

${ }^{18}$ F. Eßer et al., Appl. Phys. Lett. 107, 062103 (2015).

${ }^{19}$ I. A. Buyanova et al., Appl. Phys. Lett. 75, 501 (1999).

${ }^{20}$ A. Mintairov et al., Phys. Rev. Lett. 87, 277401 (2001).

${ }^{21}$ K. Matsuda et al., Appl. Phys. Lett. 78, 1508 (2001).

${ }^{22}$ H. P. Xin et al., Appl. Phys. Lett. 74, 2337 (1999).

${ }^{23}$ S. Francoeur et al., Appl. Phys. Lett. 75, 1538 (1999).

${ }^{24}$ I. A. Buyanova et al., Appl. Phys. Lett. 77, 2325 (2000).

${ }^{25}$ A. Patanè et al., Appl. Phys. Lett. 93, 252106 (2008).

${ }^{26}$ R. A. Mair et al., Appl. Phys. Lett. 76, 188 (2000). 
${ }^{27}$ I. A. Buyanova et al., Semicond. Sci. Tech. 17, 815 (2002).

${ }^{28}$ M. Baranowski et al., Appl. Phys. Lett. 100, 202105 (2012).

${ }^{29}$ A. Kaschner et al., Appl. Phys. Lett. 78, 1391 (2001).

${ }^{30}$ J. Bhattacharyya et al., Appl. Phys. Lett. 97, 031101 (2010).

${ }^{31}$ H. J. Polland et al., Phys. Rev. B 35, 8273(R) (1987).

${ }^{32}$ S. Sinning et al., Appl. Phys. Lett. 86, 161912 (2005).

${ }^{33}$ M. Oueslati et al., Phys. Rev. B 37, 3037 (1988).

${ }^{34}$ M. Baranowski et al., J. Phys. Condens. Mat. 23, 205804 (2011).

${ }^{35}$ C. J. Hwang, Phys. Rev. B 8, 646 (1973).

${ }^{36}$ D. Bimberg et al., Phys. Rev. B 31, 7788 (1985).

${ }^{37}$ A. Y. Cho, Thin Solid Films 100, 291 (1983).

${ }^{38}$ C. J. Hwang, J. Appl. Phys. 42, 4408 (1971).

${ }^{39}$ H. C. Casey et al., J. Appl. Phys. 44, 1281 (1973).

${ }^{40}$ G. B. Lush et al., J. Appl. Phys. 72, 1436 (1992). 